\title{
Vocal Disorders in Patients with COVID 19 in Egypt
}

\author{
Azza Abdel Aziz Azzam ${ }^{1}$ Azza Samy ${ }^{1} \cdot$ Ihab Sefein $^{2} \cdot$ Iman EIRouby $^{1,3}$ (i)
}

Received: 22 April 2021 / Accepted: 19 May 2021/Published online: 28 May 2021

(C) Association of Otolaryngologists of India 2021

\begin{abstract}
COVID-19 is considered a respiratory disease which has many symptoms associated with the larynx and the lungs infections. COVID-19 has wide spectrum of clinical features starting from mild symptoms to severe illness. Otolaryngological symptoms as nasal obstruction, loss of smell, taste dysfunction, sore throat, sticky mucus, and dysphagia are common in COVID-19 patients. Other vocal symptoms as dysphonia and phonesthenia are common in COVID-19 patients. The aim of this study is to detect the occurrence of vocal symptoms in COVID-19 patients in Egypt and to investigate the videolaryngoscopic findings associated with these symptoms. A total number of 106 patients diagnosed with COVID-19 were randomly assessed for vocal symptoms. The following epidemiological and clinical data were collected: age, gender, smoking consumption, general symptoms, otolaryngological and vocal symptoms as dysphonia and phonesthenia. Auditory perceptual assessment of voice and videolaryngoscopic examination were done. The occurrence of dysphonia and phonesthenia were observed in COVID -19 patients. Of the 106 patients, 84 patients (79\%) were dysphonic, $20(18.8 \%)$ patients were phonesthenic. The correlation of the different otolaryngological symptoms with
\end{abstract}

Iman ElRouby

imanelrouby@hotmail.com

1 Phoniatrics Department, National Hearing and Speech Institute (HSI), General Organization for Teaching Hospitals and Institutes (GOTHI), Giza, Egypt

2 Department of Otolaryngology (Ear, Nose and Throat), National Hearing and Speech Institute, General Organization for Teaching Hospitals and Institutes (GOTHI), Giza, Egypt

3 Department of Phoniatrics, Hearing and Speech Institute, 1st Tayar Fekry Street, Embaba, Giza 12651, Egypt dysphonia and phonesthenia were reported. A significant correlation was found between dysphonic patients and rhinorrhea, taste dysfunction, sore throat, and cough. A significant correlation was found between phonesthenic patients and allergic rhinitis. Videolaryngoscopic findings were detected in COVID-19 patients. Vocal fold congestion was found in 42 patients $(39.6 \%)$, benign vocal fold swellings was found in 18 patients $(16.9 \%)$, ventricular hypertrophy was found in 6 patients $(0.05 \%)$, unilateral vocal fold immobility was found in 14 patients (13.2\%), and vocal fold congestion associated with ventricular fold hypertrophy was found in 20 patients $(18.8 \%)$. There was significant correlation of dysphonia and phonesthenia with vocal fold congestion $(P$ value:0.001, $P$ value:0.039 respectively).There was a significant correlation between cough and vocal fold congestion ( $P$ value: 0.000$)$. Benign vocal fold swellings were associated with 18 patients $(16.9 \%)$, but it was not statistically significant ( $P$ value: 0.931). Dysphonia and phonesthenia were observed in patients with mild to moderate COVID-19.The vocal symptoms were associated with different laryngoscopic findings, in which, vocal fold congestion was the commonest.

Keywords COVID-19 - Dysphonia - Vocal symptoms · Otolaryngological symptoms

\section{Introduction}

Coronavirus disease- 2019 (COVID -19) is a universal pandemic declared by the World health organization that originated in Wuhan, china in late 2019 and spread globally all over the world [1]. COVID-19 is mostly considered as a respiratory disease, and many of its symptoms are 
associated with the larynx and the lungs infections [2]. COVID-19 has wide spectrum of clinical features starting from mild symptoms to severe illness which may lead to progressive respiratory failure due to alveolar damage and even death [3]. Incubation period ranges from 2 to 14 days. Common clinical features include fever, headache, cough, rhinorrhea, dyspnea, and myalgia [4]. Other less common manifestations include nausea, vomiting, or diarrhea [5].

Otolaryngological symptoms as nasal obstruction, loss of smell, taste dysfunction, sore throat, sticky mucus, and dysphagia are common in COVID-19 patients [6]. An important function of the respiratory system is to provide energy and produce phonation for the purpose of speech and communication. Voice is produced through a 3-stage process including respiration, phonation and resonation. In the respiratory stage, the force that is needed for generating sound is provided by the air expelled from the lungs. A person with the coronavirus may experience shortness of breath and may also have difficulty exhaling, which results in the lack of energy to produce sound. However, in the phonatory stage, the subglottal pressure must reach a certain point to set the vocal folds into a vibratory position. When the first stage of speech mechanism is affected, the phonation function of the larynx will be accordingly impaired causing dysphonia [7].

Dry cough also may cause changes in the vocal folds and consequently will give rise to changes in the acoustic parameters related to voice quality and may cause dysphonia. A study done by Lechien et al. using self-assessment questionnaires, reported that $26.8 \%$ of those infected with COVID-19 showed symptoms of dysphonia [8].

This study aims to detect the vocal disorders in COVID19 pandemic caused by SARS-CoV-2 patients and to detect its association with different clinical general and otolaryngological symptoms and to assess the videolaryngoscopic findings in those patients.

\section{Aim of this Study}

The aim of this study is to detect the occurrence of vocal symptoms in Egyptian COVID-19 patients and to investigate the videolaryngoscopic findings associated with these symptoms.

\section{Participants and Methods}

A total number of 106 patients diagnosed with COVID-19 were randomly assessed for vocal symptoms and enrolled in this study referred to the outpatient clinic of Otolaryngology and Phoniatrics department at the National Hearing \& Speech Institute from May 2020 to December 2020.
They were 78 females and 28 males. Their ages ranged from 18 to 66 with mean age 42.25 .

Diagnosis of COVID-19 was based on WHO interim guidance, nasopharyngeal swabs were collected in suspected patients and the virus was identified through reverse transcription polymerase chain reaction (RT-PCR) analysis and laboratory findings. Patients were referred for voice assessment after two weeks of having negative PCR results. The approval of the local ethics committee of the National Hearing and Speech Institute was obtained, and a fully written informed consent was signed by the patients before participating in the study.

\section{Inclusion Criteria}

- Adult patients (>18 years).

- Laboratory-confirmed previous COVID-19 infection (reverse transcription polymerase chain reaction, RTPCR).

- Mild to moderate cases diagnosed with COVID-19.

- Not more than 1 month have passed from the recovery of COVID-19.

\section{Exclusion Criteria}

- Patients who reported dysphonia prior to the infection with COVID -19.

- Severe cases or hospitalized cases of COVID-19.

- Patients with a history of benign or malignant laryngeal lesions, chemotherapy or radiotherapy, or history of head \& neck trauma, or previous head and neck cancer surgery.

The following epidemiological and clinical data were collected: age, gender, smoking consumption, general symptoms, otolaryngological and vocal symptoms as dysphonia and phonesthenia. Auditory perceptual assessment of voice and videolaryngoscopic examination were done.

\section{Statisical Analysis}

All statistical calculations were done using computer program IBM SPSS (Statistical Package for the Social Science; IBM Corp, Armonk, NY, USA) release 22 for Microsoft Windows. Data were statistically described in terms of mean/SD and percentages. Correlation between dysphonia, epidemiological data, general, otolaryngological symptoms and videolaryngoscopic findings were studied using Spearman rank correlation equation. Two-sided $P$ values less than 0.05 was considered statistically significant. 


\section{Results}

The epidemiological data and general, otolaryngological, and vocal symptoms from all patients (106 patients) were summarized in Table 1 and Fig. 1.They were 28 males (26\%) and 78 females (74\%). There was no significant correlation between dysphonia and phonesthenia with gender. Their ages ranged from 18 to 66 with mean age 42.25. Regarding the age, there was no significant correlation between age with dysphonia $(P$ value: 0.988$)$ and phonesthenia ( $P$ value: 0.718 ). The number of smokers were 20 , while the number of nonsmokers were 86 .There was no significant correlation between smoking with dysphonia ( $P$ value: 0.064$)$ and phonesthenia $(P$ value: 0.851$)$ in all patients.

The occurrence of dysphonia and phonesthenia were observed in COVID -19 patients. Of the 106 patients, 84 patients (79\%) were dysphonic and $20(18.8 \%)$ patients were phonesthenic (Fig. 2).

The correlation of the different otolaryngological symptoms with dysphonia and phonesthenia are clarified in Table 2. A significant correlation was found between dysphonic patients and rhinorrhea, taste dysfunction, sore throat, and cough. A significant correlation was found between phonesthenic patients and allergic rhinitis.

Videolaryngoscopic findings were summarized in Fig. 3 and examples of some videolaryngoscopic findings are presented in Fig. 4. Vocal fold congestion was found in 42 patients (39.6\%), benign vocal fold swellings was found in 18 patients $(16.9 \%)$, ventricular hypertrophy was found in 6 patients $(0.05 \%)$, unilateral vocal fold immobility was found in 14 patients $(13.2 \%)$, and vocal fold congestion associated with ventricular fold hypertrophy was found in 20 patients $(18.8 \%)$.There was significant correlation of dysphonia and phonesthenia with vocal fold congestion ( $P$ value:0.001, $P$ value:0.039 respectively) as shown in Table 3.

There was a significant correlation between cough and vocal fold congestion ( $P$ value: 0.000$)$. Benign vocal fold swellings were associated with 18 patients $(16.9 \%)$, but it was not statistically significant $(P$ value: 0.931$)$ as shown in Table 4.

\section{Discussion}

Clinical general manifestations are present in patients with Covid-19 as headache, fever, myalgia, dyspnea, cough, chest pain, abdominal pain, nausea and diarrhea. Other otolaryngological symptoms are encountered as loss of smell and taste dysfunction, nasal obstruction, rhinorrhea, sore throat and sticky mucus. The aim of this study was to detect whether there were associated voice manifestations as dysphonia and phonesthenia in covid-19 patients with mild to moderate symptoms.

Table 1 Otolaryngological and general symptoms in COVID-19 patients

\begin{tabular}{|c|c|c|c|}
\hline $\begin{array}{l}\text { Otolaryngological and general } \\
\text { symptoms }\end{array}$ & $\begin{array}{l}\text { All patients } \\
\mathrm{N}(106) 100 \%\end{array}$ & $\begin{array}{l}\text { Female } \\
\mathrm{N}(78) 73.58 \%\end{array}$ & $\begin{array}{l}\text { Male } \\
\mathrm{N}(28) 26.41 \%\end{array}$ \\
\hline Loss of smell & $76(71.69 \%)$ & $68(87.1 \%)$ & $8(28.5 \%)$ \\
\hline Nasal obstruction & $72(67.92 \%)$ & $57(73.07 \%)$ & $15(53.5 \%)$ \\
\hline Rhinorrhea & $74(69.81 \%)$ & $61(78.2 \%)$ & $13(46.4 \%)$ \\
\hline Taste dysfunction & $54(50.94 \%)$ & $50(64.1 \%)$ & $4(14.2 \%)$ \\
\hline Sore throat & $60(56.60 \%)$ & $52(66.6 \%)$ & $8(28.57 \%)$ \\
\hline Sticky mucus/phlegm & $16(15.09 \%)$ & $13(16.66 \%)$ & $3(1.7 \%)$ \\
\hline Dysphonia & $84(79.24 \%)$ & $60(76.9 \%)$ & $24(85.71 \%)$ \\
\hline Phonesthenia & $20(18.86 \%)$ & $16(20.5 \%)$ & $4(14.2 \%)$ \\
\hline Dysnea & $53(50 \%)$ & $43(55.1 \%)$ & $10(35.7 \%)$ \\
\hline Cough & $52(49.04 \%)$ & $26(33.3 \%)$ & $26(92.85 \%)$ \\
\hline Chest pain & $20(18.86 \%)$ & $11(14.1 \%)$ & $9(32.1 \%)$ \\
\hline Fever $>38$ & $48(45.2 \%)$ & $29(37.1 \%)$ & $19(67.8 \%)$ \\
\hline Myalgia & $54(50.94 \%)$ & $42(53.8 \%)$ & $12(42.8 \%)$ \\
\hline Diarrhea & $24(22.64 \%)$ & $19(24.35 \%)$ & $5(17.85 \%)$ \\
\hline Abdominal pain & $12(11.32 \%)$ & $9(11.5 \%)$ & $3(10.7 \%)$ \\
\hline Nausea, vomiting & $18(16.98 \%)$ & $14(17.9 \%)$ & $4(14.2 \%)$ \\
\hline
\end{tabular}


Fig. 1 Otolaryngological and general symptoms in COVID-19 patients

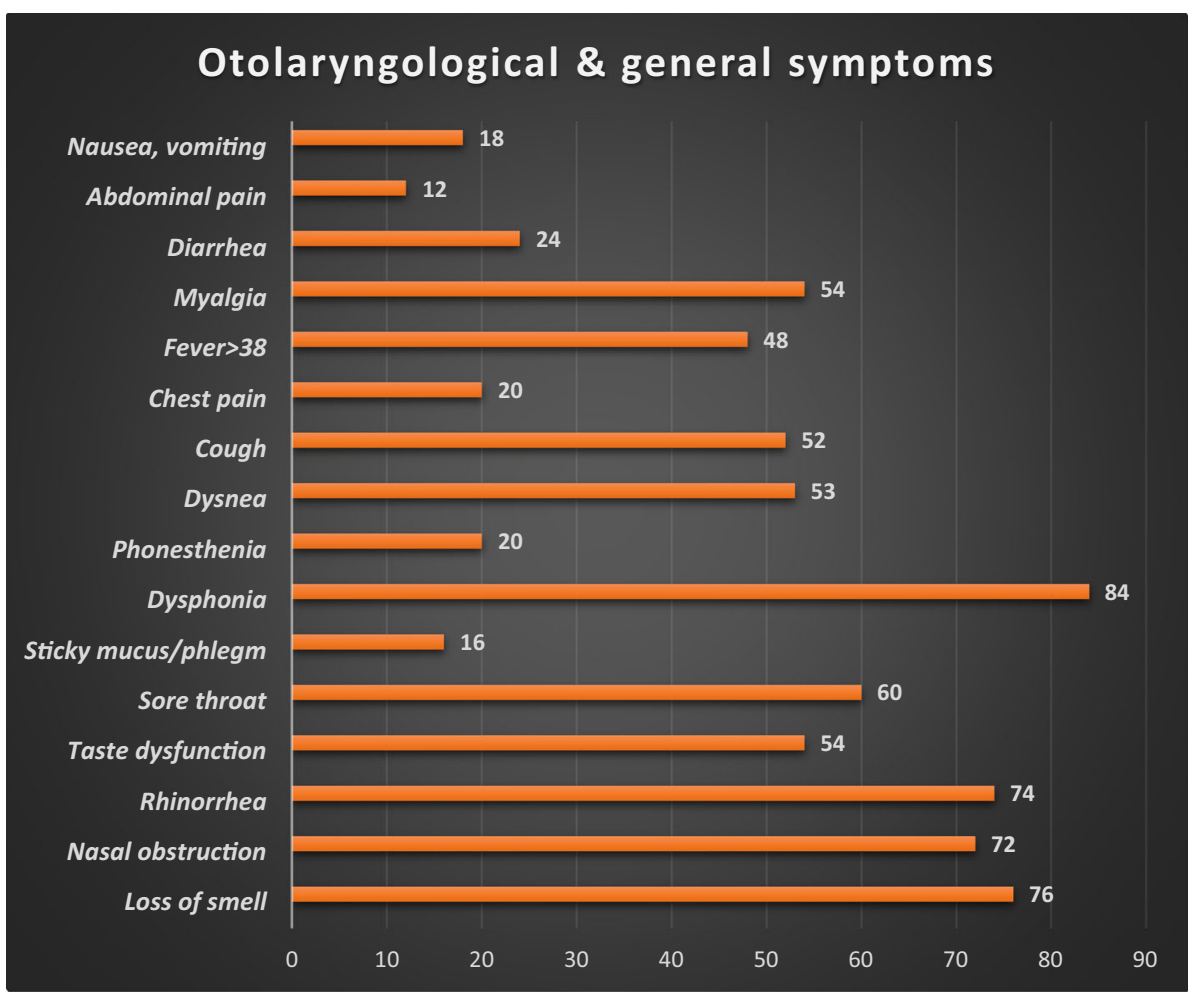

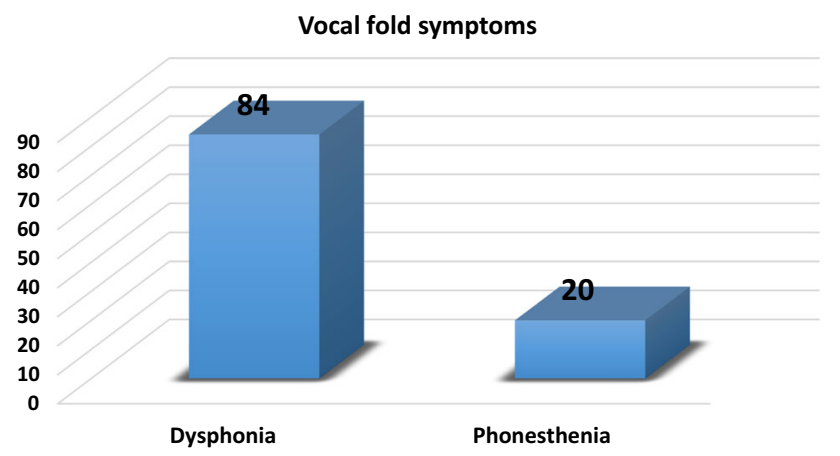

Fig. 2 Vocal fold symptoms in COVID-19 patients

The occurrence of dysphonia was observed in 84 patients $(79 \%)$ while the occurrence of phonesthenia was observed in 20 patients $(18.8 \%)$. Dysphonia may be related to laryngeal involvement by the airway inflammatory process causing vocal fold edema or inflammation [8]. Another study by Lechien et al. [7] observed that vocal folds were associated with a high expression of angiotensin-converting enzyme 2 (ACE2), which is the COVID19 receptor.

In this study, there was no correlation of age with dysphonia or phonesthenia. The number of old patients were minimal in this study, this could be related to that most of the older patients could have been infected with a more severe form of Covid presentation and had been hospitalized which excludes them from our experimental study group.

There was also no correlation of gender with dysphonia, however in other studies, females were more at risk for dysphonia [9]. In a study done by Lechien et al. [6], the female proportion was significantly higher in the dysphonic group compared to the nondysphonic group. This could be related to a gender-related difference in the inflammatory process and to the differences in the expression of ACE2 and the clinical presentation of the disease [10, 11]. Other studies detected the differences between males and females in the immune response to COVID-19 infection and inflammatory diseases. They found that females are less susceptible to have complications related to viral infections based on a different innate immunity, steroid hormones and factors related to sex chromosomes. They explained that the immune regulatory genes encoded by female $\mathrm{X}$ chromosome may cause lower viral load levels, causing less inflammation compared with male patients [12].

Videolaryngoscopic examination of the patients was used to help in the characterization of the dysphonia and phonesthenia. The different laryngoscopic findings detected were: vocal fold congestion being the most common finding, vocal fold benign swellings, vocal fold immobility, ventricular hypertrophy and vocal fold congestion associated with ventricular hypertrophy. This is consistent with the study done by Zhou et al. [3] indicated that the angiotensin-converting enzyme II (ACE2) is likely the cell 
Table 2 Correlation of otolaryngological symptoms with dysphonia and phonasthenia

\begin{tabular}{llll}
\hline Otolaryngological symptoms & & Dysphonia & Phonasthenia \\
\hline Loss of smell & Correlation coefficient & 0.092 & -0.018 \\
& $P$ value & 0.350 & 0.853 \\
Rhinorrhea & Correlation coefficient & 0.222 & 0.061 \\
& $P$ value & $0.022^{*}$ & 0.531 \\
Taste dysfunction & Correlation coefficient & 0.335 & 0.135 \\
Sore throat & $P$ value & $0.000^{* *}$ & 0.166 \\
& Correlation coefficient & 0.393 & 0.082 \\
Cough & $P$ value & $0.000^{* *}$ & 0.405 \\
Allergic rhinitis & Correlation coefficient & 0.363 & 0.154 \\
& $P$ value & $0.000^{* *}$ & 0.115 \\
& Correlation coefficient & 0.109 & 0.284 \\
\end{tabular}

*Satistically significant $P$ value $<0.05$

$* *$ Statistically highly significant $<0.001$

Fig. 3 Videolaryngoscopic findings in COVID-19 patients

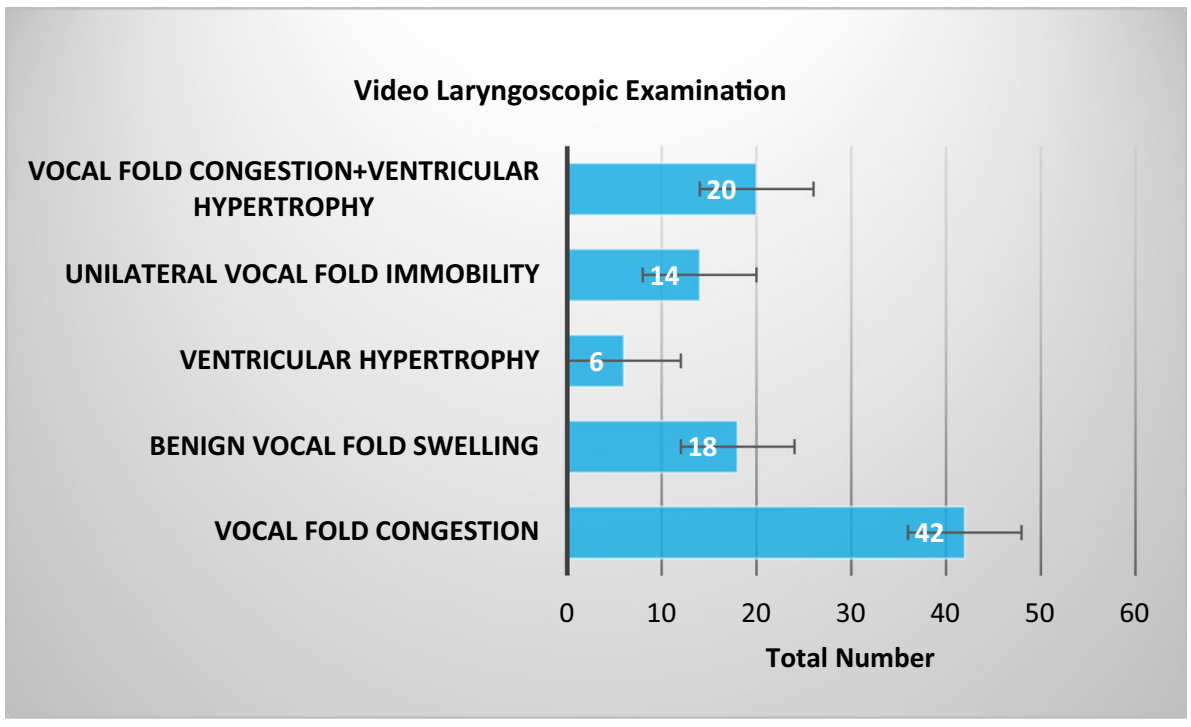

receptor of COVID -19. High ACE2 expression was identified in type II alveolar cells (AT2) of lung, esophagus upper and stratified epithelial cells, ileum and colon, myocardial cells, kidney proximal tubule cells, and bladder urothelial cells [13-16]. These findings indicated that those organs with high ACE2-expressing cells should be considered as potential high risk for COVID-19 infection. Lechien et al. reported that an ongoing study conducted in the Anatomy Department of University of Mons (Unpublished data) observed that vocal folds were associated with a high expression of ACE2. This could be an explanation to the laryngeal involvement by the airway inflammatory process causing vocal fold edema or inflammation. This hypothesis regarding the ACE2 expression in laryngeal tissue has to be investigated in future studies.

Unilateral vocal fold immobility was another finding detected in this study in 14 patients $(13.2 \%)$, however, a larger number of patients need to be investigated to find out whether this was related to the COVID-19 infection or not. From a biomolecular standpoint, viruses could infect peripheral neurons, using the cell machinery of active transport to access the central nervous system [17]. In the study of Mao et al., patients with peripheral nervous system symptoms attributed to COVID-19 infection, have reported hypogeusia $(5.6 \%)$ and hyposmia $(5.1 \%)$ [18]. Olfactory and gustatory dysfunction is also prevalent in European COVID-19 patients [19]. The ability of human coronavirus 
A

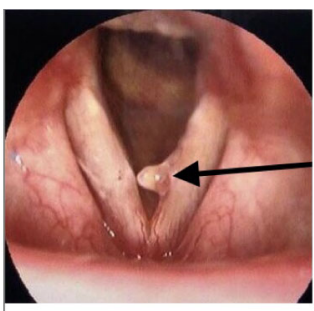

C

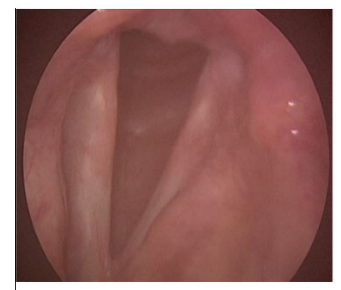

B

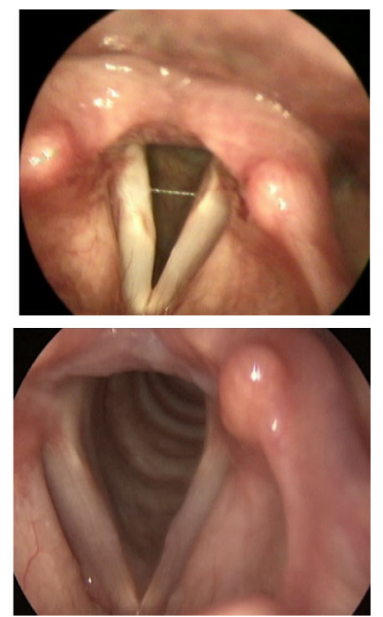

Fig. 4 a Left vocal fold polyp (black arrow), b vocal fold congestion with bilateral ventricular hypertrophy, $\mathbf{c}$ right vocal fold immobility, d vocal fold congestion

to invade the central nervous system needs future research studies to improve the knowledge about the clinical presentation of patients. Medical imaging and neuropathology will play an important role to diagnose abnormalities in olfactory bulb, cranial nerves, and brain of COVID-19 patients.

Benign vocal fold swellings were observed in some patients in this study by videolaryngoscopic examination. This could be related also to coughing as the mechanical forces of contact pressure are remarkably larger than that in normal phonation which may cause vocal fold epithelial injuries [20]. There was significant correlation between dysphonia and cough and significant correlation between cough and vocal fold congestion in the videolaryngoscopic finding. Our study agrees with the study done by Asiaee et al. [5] as they detected that there was a difference in
Table 4 correlation of videolaryngoscopic findings and cough

\begin{tabular}{lll}
\hline Videolaryngoscopic findings & & Cough \\
\hline Vocal folds congestion & $\begin{array}{c}\text { Correlation } \\
\text { coefficient }\end{array}$ & 0.363 \\
& $P$ value & $0.000^{* *}$ \\
Vocal folds swellings & $\begin{array}{c}\text { Correlation } \\
\text { coefficient }\end{array}$ & 0.009 \\
& $P$ value & 0.931 \\
Ventricual folds hypertrophy & $\begin{array}{c}\text { Correlation } \\
\text { coefficient }\end{array}$ & 0.159 \\
& $P$ value & 0.104 \\
Unilateral vocal fold immobility & $\begin{array}{c}\text { Correlation } \\
\text { coefficient }\end{array}$ & 0.048 \\
& $P$ value & 0.622 \\
Congestion\& ventricular & Correlation & 0.136 \\
hypertrophy & coefficient & 0.166 \\
& $P$ value & \\
\hline
\end{tabular}

**Statistically highly significant $<0.001$

fundamental frequency variation between the healthy and infected participants, which could result from tremor and insufficient control over laryngeal muscles. They also observed an increase in jitter and shimmer in both female and male patients. Inflammation or degeneration of the vocal fold tissues as a result of recurrent dry coughs, could explain the higher values of jitter and shimmer in the patients [21].

This study is a preliminary exploratory study that probes the occurrence of vocal symptoms as dysphonia and phonesthenia in Egyptian COVID-19 patients. Videolaryngoscopic assessment helped in the characterization of the dysphonia and phonesthenia. The study needs to be done on a larger scale of COVID-19 patients to identify the

Table 3 Correlation of videolaryngoscopic findings with dysphonia and phonasthenia

\begin{tabular}{llll}
\hline Videolaryngoscopic findings & & Dysphonia & Phonesthenia \\
\hline Vocal folds congestion & Correlation coefficient & 0.319 & 0.201 \\
& $P$ value & $0.001^{* *}$ & $0.039^{*}$ \\
Vocal folds benign swelling & Correlation coefficient & 0.046 & 0.218 \\
& $P$ value & 0.643 & 0.025 \\
Ventricular folds hypertrophy & Correlation coefficient & 0.025 & 0.014 \\
& $P$ value & 0.802 & 0.888 \\
Vocal folds immobility & Correlation coefficient & 0.075 & 0.026 \\
& $P$ value & 0.444 & 0.795 \\
Congestion\& ventricular hypertrophy & Correlation coefficient & 0.110 & 0.014 \\
& $P$ value & 0.262 & 0.887 \\
\hline
\end{tabular}

* Satistically significant $P$ value $<0.05$

**Statistically highly significant $<0.001$ 
prevalence of these vocal symptoms and to identify the associated laryngoscopic findings. The study also was focusing only on patients with mild to moderate COVID-19 presentations. Future studies may be performed on wider number of patients with different clinical presentations.

\section{Conclusion}

Dysphonia and phonesthenia were observed in patients with mild to moderate COVID-19 disease.Vocal symptoms were associated with different laryngoscopic findings, in which, vocal fold congestion was the commonest. Future epidemiological and clinical studies are required to investigate the associated vocal symptoms and the videolaryngoscopic findings in COVID-19 patients and to explore the underlying cause of these symptoms.

\section{References}

1. Zu ZY et al (2020) Coronavirus disease 2019 (COVID-19): a perspective from China. Radiology 296(2):E15-E25. https://doi. org/10.1148/radiol.2020200490

2. Van der Hoek L et al (2004) Identification of a new human corona virus. Nat Med. 10(4):368-373

3. Zhou P et al (2020) A pneumonia outbreak associated with a new coronavirus of probable bat origin. Nature. https://doi.org/10. 1038/s41586-020-2012-7

4. Wang, D. et al (2020) Clinical characteristics of 138 hospitalized patients with 2019 novel coronavirus-infected pneumonia in Wuhan, China. JAMA. https://jamanetwork.com/journals/jama/ fullarticle/2761044 .

5. Kooraki S et al (2020) Coronavirus (COVID-19) outbreak: what the department of radiology should know. J Am Coll Radiol 17:447-451

6. Lechien JR et al (2020) Clinical and epidemiological characteristics of 1420 European patients with mild-to-moderate coronavirus disease 2019. J Intern Med 288:335-344

7. Asiaee M, Vahedian-azimi A, Atashi SS, Keramatfar A, Nourbakhsh M (2020) Voice quality evaluation in patients with COVID-19: an acoustic analysis. J Voice. https://doi.org/10. 1016/j.jvoice.2020.09.024

8. Lechien JR, Chiesa-Estomba CM, Cabaraux P et al (2020) Features of mild to-moderate COVID-19 patients with dysphonia. J Voice. https://doi.org/10.1016/J.JVoice.2020.05.012

9. Buselli R, Corsi M, Necciari G, Pistolesi P, Baldanzi S, Chiumiento M, Del Lupo E, Del Guerra P, Cristaudo A (2020) Sudden and persistent dysphonia within the framework of COVID-19: the case report of a nurse. Brain Behavior Immun Health. https://doi.org/10.1016/j.bbih.2020.100160

10. La Vignera S, Cannarella R, Condorelli RA et al (2020) Sexspecific SARS-CoV-2 mortality: among hormone-modulated ACE2 expression, risk of venous thromboembolism and hypovitaminosis D. Int J Mol Sci. https://doi.org/10.3390/ ijms21082948

11. Chamekh M, Deny M, Romano M et al (2017) Differential susceptibility to infectious respiratory diseases between males and females linked to sex-specific innate immune inflammatory response. Front Immunol 13(8):1806

12. Conti P, Younes A (2020) Coronavirus COV-19/SARS-CoV-2 affects women less than men: clinical response to viral infection. J Biol Regul Homeost Agents https://doi.org/10.23812/ Editorial-Conti-3

13. Zou X et al (2020) The single-cell RNA-seq data analysis on the receptor ACE2 expression reveals the potential risk of different human organs vulnerable to Wuhan 2019-nCoV infection. Med Front. https://doi.org/10.1007/s11684-020-0754-0

14. Zhao Y et al (2020) Single-cell RNA expression profiling of ACE2, the putative receptor of Wuhan 2019-nCov. Preprint at https://www.biorxiv.org/content/ https://doi.org/10.1101/2020.01.26.919985v1.

15. Zhang $\mathrm{H}$ et al (2020) The digestive system is a potential route of 2019-nCov infection: a bioinformatics analysis based on singlecell transcriptomes. Preprint at https://www.biorxiv.org/content/ https://doi.org/10.1101/2020.01.30.927806v1

16. Chai $X$ et al (2020) Specific ACE2 expression in cholangiocytes may cause liver damage after 2019-nCoV infection. Preprint at https://www.biorxiv.org/content/ https://doi.org/10.1101/2020.02.03.931766v1.

17. Koyuncu OO, Hogue IB, Enquist LW (2013) Virus infections in the nervous system. Cell Host Microbe 13(4):379-393. https://doi.org/10.1016/j.chom.2013.03.010

18. Mao L, Wang M, Chen S et al (2020) Neurological manifestations of hospitalized patients with COVID-19 in Wuhan, China: a retrospective case series study. MedRXiv . https ://doi.org/ https://doi.org/10.1101/2020.02.22.20026500

19. Lechien LR et al (2020) Olfactory and gustatory dysfunctions as a clinical presentation of mild-to-moderate forms of the coronavirus disease (COVID-19): a multicenter European study. Eur Arch Otorhinolaryngol 277:2251-2261. https://doi.org/10.1007/ s00405-020-05965-1

20. Hess MM, Verdolini K, Bierhals W et al (1998) Endolaryngeal contact pressures. J Voice 12:50-67

21. Ferrand CT (2012) Voice disorders: scope of theory and practice. Allyn and Bacon, Boston

Publisher's Note Springer Nature remains neutral with regard to jurisdictional claims in published maps and institutional affiliations. 